\title{
The effect of synthetic alginate sulfate hydrogels with recombinant PDGF-BB on Wound healing
}

\author{
Babavalian $\mathrm{H}^{1,2}$, Tebyanian $\mathrm{H}^{2,3}$, Latifi $\mathrm{AM}^{2}$, Shokrgozar $\mathrm{MA}^{4}$, Bonakdar $\mathrm{S}^{4}$, Shakeri $\mathrm{F}^{2}$ \\ Applied Virology Research Center, Baqiyatallah University of Medical Sciences, Tehran, Iran. \\ babavalianh@gmail.com
}

\section{ABSTRACT}

OBJECTIVE: Platelet-derived growth factor (PDGF-BB) is an important factor in the regeneration and wound healing. One of the problems is that there is not enough efficiency in the transmission or delivery of such factors in the wound site. In this study, alginate sulfate hydrogel was synthesized with recombinant PDGF-BB growth factor for achieving a quick method in wound healing.

METHODS: In this study, Alginate sulfate hydrogel was made by photo-crosslinking method and methacrylate. Its toxicity was evaluated by MTT assay. Transforming growth factor was studied in releasing from synthesized alginate sulfate hydrogels and also, lack of toxicity was confirmed, and hydrogel was made with a recombinant human growth factor. Wounds were created with a diameter of $10 \mathrm{~mm}$ on the back of rats in order to check the wound healing.

RESULTS: This study showed that alginate sulfate hydrogels-PDGF-BB were faster in wound healing than non-sulfate alginate hydrogels-PDGF-BB. Therefore, the controlled delivery of growth factor system can be a powerful idea for therapeutic applications for wound healing.

CONCLUSION: Alginate sulfate hydrogel with recombinant growth factor $4 \mu \mathrm{g} / \mathrm{cm}^{2}$ (PDGF-BB) was very suitable for wound healing (Tab. 1, Fig. 3, Ref. 34). Text in PDF www.elis.sk.

KEY WORDS: alginate hydrogel, PDGF-BB, wound healing.

\section{Introduction}

Skin wounds and tissue damage are important issues in healing. The importance of this issue is especially significant during disasters and crisis conditions, which need appropriate treatment methods and to promote healing of injuries $(1,2)$. Wound dressing material is placed directly on the surface of the wound and it should be designed in an appropriate way, which improves various stages of healing. Modern dressings have different types and different classifications. Natural hydrogels are the most common types of modern wound dressings, and can be used as carriers for targeted compounds to accelerate wound healing (2-5). The natural hydrogel can be widely used in the preparation of modern wound healing, such as alginic acid. The alginic acid polymer is a structural component, which can be obtained from polysaccharide cell wall (6). Alginate has many properties, such as: nontoxic, non-carcinogenic, non-allergic, absorptive, a hemostatic

\footnotetext{
${ }^{1}$ Applied Virology Research Center, Baqiyatallah University of Medical Sciences, Tehran, Iran, ${ }^{2}$ Department of Tissue Engineering and Regenerative Medicine, Nanobiotechnology Research Center, Baqiyatallah University of Medical Sciences, Tehran, Iran, ${ }^{3}$ Research Center for Prevention of Oral and Dental Disease, Baqiyatallah University of Medical Sciences, Tehran,Iran, and ${ }^{4}$ National Cell Bank of Iran, Pasteur Institute of Iran, Tehran, Iran

Address for correspondence: H. Babavalian, Applied Virology Research Center, Baqiyatallah University of Medical Sciences, Tehran, Iran. Phone: +98.21.88617712, Fax: +98.21.82482549
}

agent, strength, biocompatibility, sterility, customizable, low-cost technology and etc. These types of dressings are more efficient in wound healing than others. In addition, the physical and chemical properties depend on calcium ions, sodium alginate hydrogels, relative concentrations, structure monomers. A rich dressing- gluconic acid ion easily reacts to sodium and produces gels with a high durability. Sodium alginate solubility causes swelling of fiber by ion exchange on the wound's surface. It is a convenient feature for wet dressings formation (7). On the other hand, better understanding and identifying of molecular and cellular mechanisms, which are involved in the healing wounds process, helps to find the best way for wound healing. Numerous studies have shown beneficial effects of these growth factors and cytokines in wound healing. Platelet-derived growth factor (PDGF-BB) is the first growth factor with chemotactic properties, which is applied for cells migration such as: neutrophils, monocytes and fibroblasts and to intensify fibroblast proliferation, extracellular matrix produced by these cells and to stimulate fibroblasts into myofibroblasts phenotype and induction of collagen matrix in the wound site (8-11). The most important usage problem of this growth factor is an inadequate delivery of growth factor in wound site and that they have a live and clinical limitation as well. So, the gradual development of a delivery system leads to healing in the shortest time. There are different strategies of growth factor delivery from hydrogels, which include direct and indirect methods. Direct delivery occurs through physical enclosed, non-covalent bonding and covalent bonding via a hydrolytic or biodegradable enzyme. An 
indirect method is based on management delivery system for gene therapy and cell transplantation (12-14). The controlled release of the growth factor PDGF-BB of alginate hydrogels is prepared by affinity or desire, which can be used in wound healing in animal models $(13,15)$. In this study, alginate sulfate hydrogel was synthesized with recombinant growth factor PDGF-BB for achieving a quick method in wound healing.

\section{Methods and materials}

\section{Alginate sulfate synthesis}

Various methods were used for sulfate sodium alginate such as: Huang Ronghna and colleagues in 2003 (16), Lihong Fan and colleagues in 2011 (17). The most important method of sulfate alginate synthesis was modified by Fang and Jiang (18). 10 gr of sodium alginate (Merck, Germany) was added to a sulfating reagent containing $80 \mathrm{~mL}$ of Formamide and $20 \mathrm{~mL}$ of Chlorosulfonic acid for synthesizing the alginate sulfate; the mixture was maintained at $60{ }^{\circ} \mathrm{C}$ for 4 hours until the mixture color turned brown. Then, $200 \mathrm{~mL}$ of acetone was added to the solution sediment and the sediment was dissolved in distilled water. The $\mathrm{pH}$ was set to $10-11$, and the solution was dialyzed and concentrated for 72 hours. Finally, the structure and amount of the sulfate hydrogel were characterized by Elemental Analyzer (Eltra CS800 ) Fourier Transform Infrared (FTIR) (PerkinElmer, USA) and nuclear magnetic resonance (NMR) (Bruker, Germany) spectroscopy (1).

\section{Synthesized alginate hydrogels sulfate by photocrosslink}

Alginate sulfate $(2.5 \%)$ was completely dissolved in $20 \mathrm{ml}$ of water. Then, $20 \mathrm{ml}$ methacrylate $(\mathrm{pH}=7)$ was added and the resulting solution was kept for 72 hours at room temperature (foil wrapped around it). After 72 hours, $40 \mathrm{ml}$ was divided into the two tubes of $50 \mathrm{ml}$ and then, $30 \mathrm{ml}$ ethanol was added to each and they were centrifuged in $1400 \mathrm{~g}$ for 5 minutes. The solvent was discarded, and the sediment was obtained. $5 \mu 1$ EDC ([N-ethyl$\mathrm{N}$ '-(dimethyl aminopropyl) carbodiimide) (0.005 in $\left.100 \mathrm{ml} \mathrm{H}_{2} \mathrm{O}\right)$ was added to $0.25 \mathrm{~g}$ alginate sulfate methacrylate powder, which was dissolved in $200 \mu$ of water, and finally, they were light up for 15-10 minutes (19).

Recombinant human platelet-derived growth factor - hydrogel synthesis

Recombinant human platelet-derived growth factor-PDGFBB $(4 \mu \mathrm{g} / \mathrm{ml})$ was added into alginate and alginate sulfate methacrylate in $1.5 \mathrm{ml}$ of water $0.05 \%$ (photoinitiator). Both solutions were mixed for 5 minutes and 300 microliters of the solution were poured in 96 tissue culture plates with $365 \mathrm{~nm}$ light was done for 10 minutes to form the hydrogel photo-crosslink. Then, each of hydrogel photo-crosslink was incubated in $15 \mathrm{ml}$ tube containing $10 \mathrm{ml}$ of phosphate salt buffer with $\mathrm{pH}=7.4$ and at $37^{\circ} \mathrm{C}$. The supernatant was removed, and the fresh buffer was full again at predetermined intervals during the three weeks. Sandwich ELISA method was used to investigate the protein concentration in the supernatant. Sample's absorption was read at $450 \mathrm{~nm}$ by ELISA reader. The growth factors present in each sample calibration was curved based on the standard serial dilutions of each and OD was plotted and the protein concentration was measured by comparing the OD values, which were obtained with the standard curve $(20,21)$.

\section{Experimental groups and design}

Rats (Albino-N-Mari, Male, 180-200 g)) were randomly divided into 3 experiment groups (each group contains 5 animals) and they were kept in individual cages. Rats were controlled in a standard environment (humidity and temperature) on a 12/12 hours light/dark cycle with standard food and water, following experimental procedures approved by the Ethical Committee for Animal Research of Baqiyatallah University of Medical Sciences. After skin preparation, the anesthesia was done with Xylene (6.5 $\mathrm{mg} / \mathrm{kg}$ ) and Ketamin $(34.5 \mathrm{mg} / \mathrm{kg})$. Dorsal incisional wounds were made with a $1 \times 1 \mathrm{~cm}$ square wound area. In this study, the treatments including Group A: control (no treatment), Group B: alginate sulfate hydrogels without recombinant growth factor, Group C: alginate ammonium hydrogels with $4 \mu \mathrm{g} / \mathrm{cm}^{2}$ recombinant growth factor. Rats were sacrificed and their wound sites were taken for microscopic studies and histology on days 4, 7, 14 and 21 (10, 22). The microscope sections were stained by Hematoxylin and Eosin.

\section{Evaluation of wound healing process}

Photos of wound sites were taken every day and were analyzed and evaluated by Image J software. In microscopy, the histological study was carried out. For histological studies, the number of rats was selected and in case the package containing chloroform were sacrificed and wound samplings were taken, the slides were stained by Hematoxylin-Eosin staining. Samples were evaluated under a light microscopy. The separation time remaining scabs and scar sizes analysis were conducted based on the photos. All the steps on animals, such as ulcers, its treatment and level measurement were performed by a certain person (23-25).

\section{Statistical analysis}

SPSS software was used to analyze data and the non-parametric Kruskal-Wallis test was used due to the low sample size in each group for comparing the target groups and Mann-Whitney was used for comparing the two groups and also, the level of statistically significant difference was considered $(\mathrm{p}<0.05)$.

\section{Results}

\section{Sulfated sodium alginate}

Sulfated alginate was achieved, and it took more time and the amount of sulfate reached to $0.6 \%$. It was confirmed by Elemental Analysis and FTIR test, that the amount was too low and we changed and improved the Protocol. Finally, $13 \%$ of sulfate was taken, which was the appropriate amount.

\section{Characterization of methacrylate alginate sulfate and photo- crosslinking hydrogel}

Methacrylate was connected to the main chain reactive carboxylic acid groups by alginate sulfate with alginate Amin (2-amino 


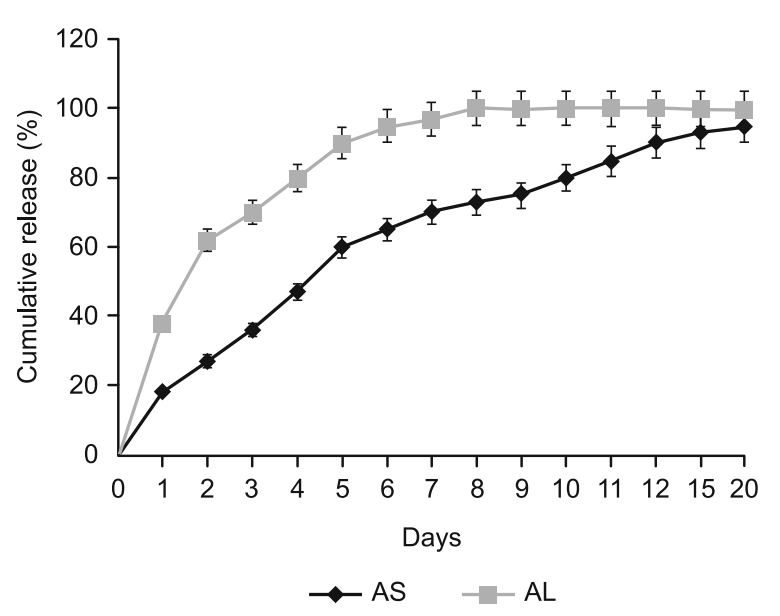

Fig. 1. Releasing Platelet-derived growth factor from alginate and sulfated alginate. Time-dependent Release of Peptide from Alginate (AL) and Alginate Sulfate (AS) Hydrogels.

ethyl Methacrylate, AEMA) for integration of alginate hydrogel into photocrosslinking alginate sulfate during the process of photo-polymerization. A stable Amide connection was paired by the chemical composition of water Carbodiimide. H-NMR spectrum of alginate sulfate methacrylate showed methylene vinyl, methylene and methylene protons, which were newly formed by reaction with AEMA and they were in the area of $\delta 6 / 2$ and 5/7, 2/9 and $1 / 95$, respectively. Spectra showed that alginate sulfate has successfully become methacrylate. Photocrosslink with H-NMR was investigated, and the emergence of peak showed methylene vinyl, which changed to $\delta 1 / 2 \mathrm{H}-\mathrm{NMR}$ spectrum. Methacrylate showed new peaks of formed methylene protons end photocrosslinking appeared at $\delta 2 / 25$. Photocrosslink alginate hydrogel discs were synthesized by using alginate and alginate sulfate. Pure morphology discs of alginate hydrogel and alginate sulfate were in distilled water. The results showed no significant difference between the groups in the pure morphology and the size of hydrogel and they were not changed after 24 hours.

Kinetics of releasing of platelet-derived growth factor from hydrogel

For the finding that" Alginate sulfate hydrogel can delay the release of growth factors through affinity binding interactions during the process UV crosslink", releasing Platelet-derived growth factor-BB was determined with using the assay from photo-crosslink alginate sulfate hydrogel in PBS buffer and was compared with photocrosslinking alginate hydrogels. Releasing growth factor was faster in alginate hydrogels than alginate sulfate hydrogel. 82 to $100 \%$ released in the first days of 5 to 7 days. On the contrary, growth factor continued to release from the alginate sulfate hydrogel over 3 weeks (Fig. 1).

\section{Macroscopic evaluation}

Evaluation of wound scabs fall time, wound healing and remaining of scar size

In this case, alginate hydrogel with $4 \mu \mathrm{g} / \mathrm{cm}^{2}$ standard recombinant growth factor $(C)$ (6-9 days) were the minimum time required for falling scab. This time for groups (A), (B), (C) was 14-20, 9-14 and 6-9 days, respectively (results based on the independent third test was the same $(p<0.05))$. Failing time of scab in the group $\mathrm{C}$ was shorter than in the other groups. Therefore, recombinant growth factor in alginate sulfate hydrogel caused the scabs to fall and wound to heal in shorter time than in the other groups. Alginate sulfate hydrogel group with $4 \mu \mathrm{g} / \mathrm{cm}^{2}$ standard
A

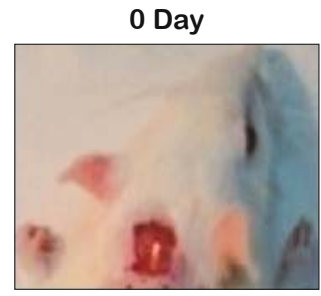

B

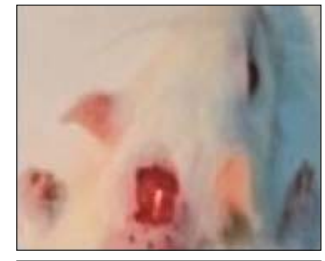

c

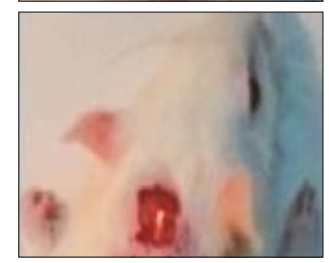

4 Days
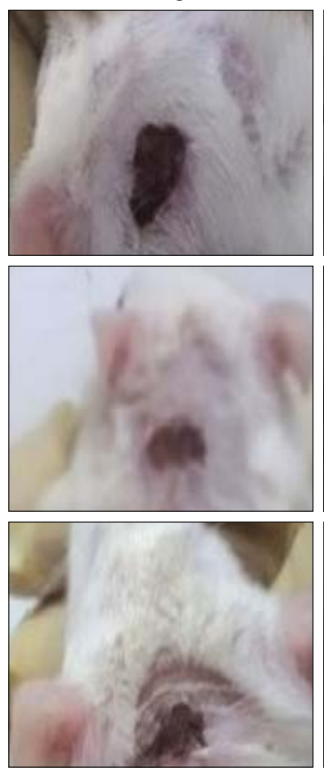

7 Days
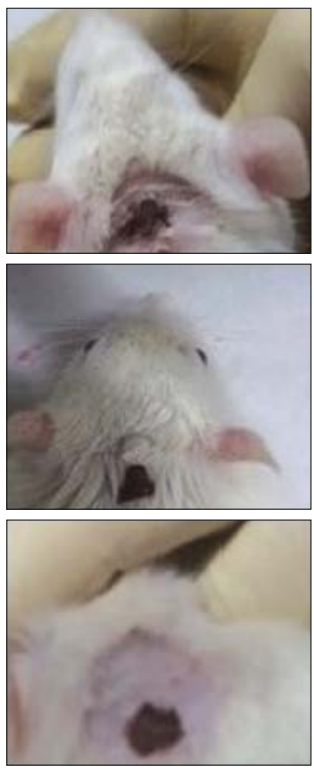

14 Days
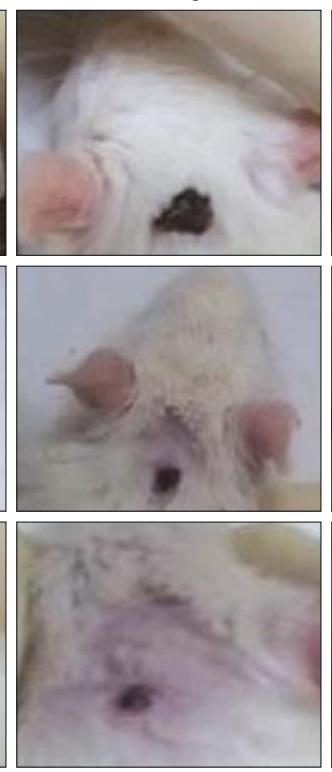

21 Days

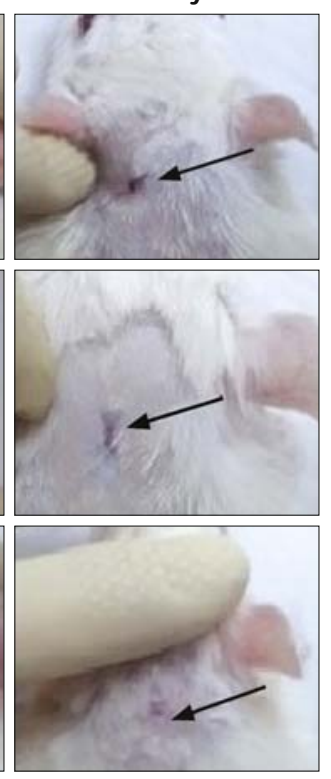

Fig. 2. Treatment between the three groups. 
A
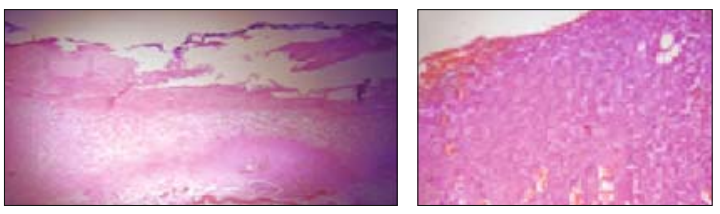

B
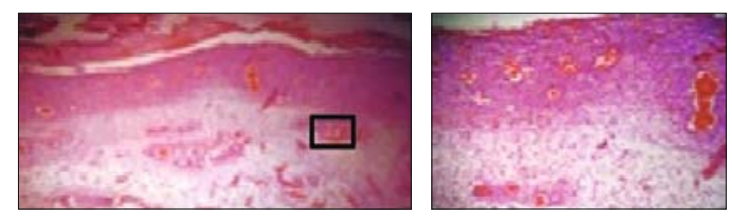

C
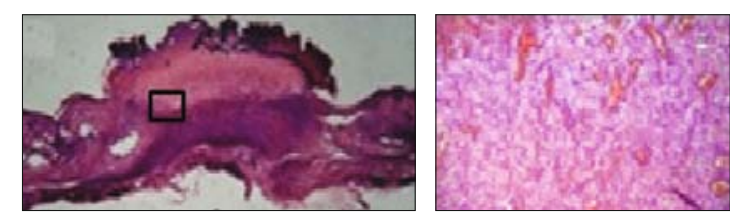

Fig. 3. Transverse sections of skin showed the vascularization with Hematoxylin-Eosin staining test in 7 days. negative control group (without treatment) (A), Alginate hydrogels sulfate without recombinant growth factor (B), Alginate hydrogels sulfate with $4 \mu \mathrm{g} / \mathrm{cm}^{2}$ growth factor (C).

recombinant growth factor had $3.1 \mathrm{~cm}^{2}$ of scarring. These amounts in the groups A, B and C were $7.2,3.5$ and $3.1 \mathrm{~cm}^{2}$, respectively (Results based on three tests and independent $(\mathrm{p}<0.05)$ ) (Fig. 2).

\section{Microscopic evaluation}

Evaluation of angiogenesis and number of fibroblasts

The results showed that the peak of vascularization was on day 7 and the group $C$ had the highest vascularization, which was a significant difference compared to all the other groups. Also, the group B showed significant differences compared to the group A (Results based on independent and three tests $(\mathrm{p}<0.05))$. Transverse sections of skin showed the vascularization with Hematoxylin-Eosin staining, they are shown in Figure 3. Results showed that the number of fibroblasts had significant differences in the experimental groups on days 4,7 and 14 and no significant differences on day 21 . The greatest increase in the number of fibroblasts was on day 14. Group C had the greatest number and had significant differences compared to all the other groups. Group B showed significant differences compared to group A as well (Results based on independent and three tests $(p<0.05))$ (Tab. 1).

Evaluation of collagen, epidermis, inflammation and hair follicle in wound healing

Sampling was performed on days 4, 7, 14 and 21. The results of microscopic studies such as: evaluating of density of hair follicles, epidermis depth, collagen and inflammation based on the presence of inflammatory cells showed that a statistically significant difference was not identified between the groups $\mathrm{C}$ and $\mathrm{B}$ (Tab. 1). The measurement of collagen showed a significant statistical difference in each group on days 4, 7, and 14 and there was not a significant statistical difference on day 21 . The greatest growth and the presence of collagen were on day 14 and the group $\mathrm{C}$ had the highest (Results based on independent and three tests $(p<0.05)$ ). Measurement of wound epidermis is an effective strategy in the amount of wound healing. The epidermal thickness was not calculated for 4 days, because the epidermis was not fully formed and was not measurable as well. After the formation of the epidermis, the size of the epidermis was different in the groups $B$ and $C$ compared to the group A on days 7 and 14 (Results based on independent and three tests $(p<0.05))$. One of the quality criteria in wound healing is the regrowth of hair follicles. According to the results, follicular growth was not seen on day 4,7 and 14, but hair follicle was seen on day 21 . The average number of follicles was observed in groups (A), (B), (C) consisting of 1,2 and 6, respectively (results based on independent and three tests $(p<0.05)$ ).

\section{Discussion}

Wound healing is a complex process, which includes the return of damaged cellular structures and tissue layers in accordance with the original and normal tissue (26). Wound healing process is completed by 3 stages: inflammation, cell proliferation and reconstruction phase (27). Although these steps are associated with each other, they can overlap in time. So, a combination can shorten the time of these phases, which naturally leads to the wound

Tab. 1. Statistical comparison of the density of hair follicles, numeric density of blood vessels, and density of collagen fibers in each group on days $4,7,14$, and 21 .

\begin{tabular}{cccccccc}
\hline Group & Day & Angiogenesis & Fibroblasts & Hair follicle & Epidermis & Collagen & Inflammation \\
\hline & 4 & $0 \pm 0$ & $224 \pm 14.01$ & $0 \pm 0$ & $0 \pm 0$ & $0 \pm 0$ & $3 \pm 0.68$ \\
A & 7 & $1.12 \pm 0.02$ & $1290 \pm 23.1$ & $0 \pm 0$ & $0 \pm 0$ & $0.22 \pm 0.0002$ & $3 \pm 0.68$ \\
& 14 & $1.50 \pm 0.1$ & $2900 \pm 31.2$ & $0 \pm 0$ & $0 \pm 0$ & $0.49 \pm 0.0023$ & $2.80 \pm 0.99$ \\
& 21 & $1.10 \pm 0.15$ & $1036 \pm 25.7$ & $1 \pm 0.0004$ & $21 \pm 1.01$ & $0.31 \pm 0.0043$ & $2.60 \pm 0.456$ \\
\hline & 4 & $0 \pm 0$ & $592 \pm 8.90$ & $0 \pm 0$ & $0 \pm 0$ & $0 \pm 0$ & $3 \pm 0.68$ \\
B & 7 & $1.15 \pm 0.45$ & $4150 \pm 19.40$ & $0 \pm 0$ & $12 \pm 1.33$ & $0.85 \pm 0.0012$ & $3 \pm 0.68$ \\
& 14 & $1.75 \pm 0.009$ & $6050 \pm 45.6$ & $0 \pm 0$ & $25 \pm 2.10$ & $1.39 \pm 0.0124$ & $2.39 \pm 0.657$ \\
& 21 & $1.55 \pm 0.001$ & $3710 \pm 20.03$ & $2 \pm 0.67$ & $57 \pm 2.66$ & $0.97 \pm 0.0456$ & $0.80 \pm 0.00067$ \\
\hline & 4 & $4 \pm 0.11$ & $5600 \pm 19.77$ & $0 \pm 0$ & $0 \pm 0$ & $1.70 \pm 0.06$ & $2.70 \pm 0.502$ \\
C & 7 & $8.10 \pm 0.160$ & $19700 \pm 49.30$ & $0 \pm 0$ & $32 \pm 1.34$ & $2.90 \pm 0.003$ & $1.80 \pm 0.609$ \\
& 14 & $6.87 \pm 0.29$ & $30100 \pm 60.22$ & $0 \pm 0$ & $145 \pm 6.09$ & $4.60 \pm 1.01$ & $0.90 \pm 0.00045$ \\
& 21 & $2.20 \pm 0.87$ & $7100 \pm 28.55$ & $6 \pm 0.1$ & $187 \pm 15.23$ & $4.43 \pm 0.921$ & $0.30 \pm 0.00007$ \\
\hline
\end{tabular}

Group A: control (no treatment), Group B: alginate hydrogels sulfated without recombinant growth factor, Group C: alginate ammonium hydrogel with $4 \mu \mathrm{g} / \mathrm{cm}^{2}$ recombinant growth factor.*Results based on the independent third test are the same $(\mathrm{p}<0.05)$ 
healing. Various studies have been done on wound healing and different materials were prepared and presented. The composition of recombinant platelet-derived growth factor PDGF-BB can be used in alginate sulfate hydrogel to a topical formulation for wound healing in some conditions such as ulcers (10). Plateletderived growth factor (PDGF-BB) is a glycoprotein with a weight of approximately $35 \mathrm{kDa}$, which contains two polypeptide chains linked together by five disulfide bonds. PDGF-BB has an important role in the pathogenesis of strong mitogenic factors, chemotactic and effective role in wound healing. PDGF-BB compounds were prescribed as gel formulation. The gel formulation of rPDGF has been approved (brand Regranex) for the treatment of rPDGF Fullthick in diabetic patients (28). Hang et al (2006) (29) evaluated the effects of PDGF-BB/CBD on cellularization and angiogenesis in collagen scaffolds. Sun et al (2007) (30) studied the effects of collagen membrane CBD-PDGF-BB in accelerating ischemic wound healing on a rabbit model. The use of alginate hydrogel is recommended according to the characteristics of biocompatibility, non-immunogenicity and the ability to form a strong gel that allows encapsulation of fragile molecules, such as peptides and proteins in its active form (31-33). The macromolecular drug is located in a hydrophilic calcium alginate chains network of alginate hydrogel and its releasing will be regulated through the permeable hydrogel matrix $(31,34)$. The releasing of this composition is affected by the breakdown of non-specific binding ions between the positively charged and negatively charged alginate growth factors and growth factors finally released in wound sites. This often leads to an unpredictable delivery of the growth factors from alginate hydrogels. In 2008, Freeman et al, evaluated the effects of alginate sulfate hydrogels for the controlled release of proteins and specific binding to heparin (34). In 2011, Jeon et al (20) evaluated the possibility of transferring growth factors based on the affinity to alginate hydrogel heparin, which is made by light and the results suggested that affinity was intended to be more sulfate rather than protein and the protein releasing was slower and it could control the releasing. On the other hand, heparin had side effects on the location of the wound. Then, it is better to use alginate sulfate hydrogels instead of alginate hydrogels heparin. In this study, the effect of alginate sulfate hydrogels containing growth factor PDGF-BB was investigated and we tried to modify the method of sulfated alginate chains for controlling the growth factor releasing. In this experiment, $13 \%$ of sulfate was obtained using laboratory techniques. The properties of alginate sulfate hydrogel were investigated with recombinant growth factor or without it in wound healing. This finding suggested that alginate sulfate hydrogel with growth factor was better than without growth factor in wound healing. Thus, alginate sulfate hydrogel with a recombinant growth factor accelerated wound healing and improved wound healing. It can be concluded that alginate sulfate hydrogel with recombinant growth factor $4 \mu \mathrm{g} / \mathrm{cm}^{2}$ (PDGF-BB ) was very suitable for the treatment of superficial wounds and dressing. Heparin protein transport systems have been used by other researchers, but in this research, PDGF-BB protein was used as a protein transport systems in wound healing.

\section{References}

1. Babavalian H, Latifi AM, Shokrgozar MA, Bonakdar S, Mohammadi S, Moghaddam MM. Analysis of Healing Effect of Alginate Sulfate Hydrogel Dressing Containing Antimicrobial Peptide on Wound Infection Caused by Methicillin-Resistant Staphylococcus aureus. Jundishapur J Microbiol 2015; 8 (9): e28320.

2. Boateng JS, Matthews KH, Stevens HNE, Eccleston GM. Wound healing dressings and drug delivery systems: a review. J Pharm Sci 2008; 97 (8): 2892-2923.

3. Pereira RF, Mendes A, Bártolo PJ. Novel alginate/aloe vera hydrogel blends as wound dressings for the treatment of several types of wounds. Chem Eng Trans 2013; 32: 1009-1014.

4. Sweeney IR, Miraftab M, Collyer G. A critical review of modern and emerging absorbent dressings used to treat exuding wounds. Int Wound J 2012; 9 (6): 601-612.

5. Watson NFS, Hodgkin W. Wound dressings. Surgery (Oxford) 2005; 23 (2): 52-55.

6. Lee KY, Mooney DJ. Alginate: properties and biomedical applications. Prog Polym Sci 2012; 37 (1): 106-126.

7. Nazeri S, Ardakani EM, Babavalian H, Latifi AM. Evaluation of Effectiveness of Honey-Based Alginate Hyrogel on Wound Healing in a Mouse Model of Rat. Journal of Applied Biotechnology Reports 2015; 2 (3): 293-729.

8. Andrae J, Gallini R, Betsholtz C. Role of platelet-derived growth factors in physiology and medicine. Genes Dev 2008; 22 (10): 1276-1312.

9. Augst AD, Kong HJ, Mooney DJ. Alginate hydrogels as biomaterials. Macromol Biosci 2006; 6 (8): 623-633.

10. Li H, Fu X, Zhang L, Huang Q, Wu Z, Sun T. Research of PDGF$\mathrm{BB}$ gel on the wound healing of diabetic rats and its pharmacodynamics. J Surg Res 2008; 145 (1): 41-48.

11. Rozman P, Bolta Z. Use of platelet growth factors in treating wounds and soft-tissue injuries. Acta Dermatovenerol APA 2007; 16 (4): 156.

12. Hiemstra C, Zhong Z, van Steenbergen MJ, Hennink WE, Feijen J. Release of model proteins and basic fibroblast growth factor from in situ forming degradable dextran hydrogels. J Control Release 2007; 122 (1): 71-78.

13. Lin C-C, Anseth KS. PEG hydrogels for the controlled release of biomolecules in regenerative medicine. J Pharm Res 2009; 26 (3): 631-643.

14. Karami A, Tebyanian H, Goodarzi V, Shiri S. Planarians: an in vivo model for regenerative medicine. Int J Stem Cells 2015; 8 (2): 128-133.

15. Silva AKA, Richard C, Bessodes M, Scherman D, Merten O-W. Growth factor delivery approaches in hydrogels. Biomacromolecules 2008; 10 (1): 9-18.

16. Ronghua H, Yumin D, Jianhong Y. Preparation and in vitro anticoagulant activities of alginate sulfate and its quaterized derivatives. Carbohydr Polym 2003; 52 (1): 19-24.

17. Fan L, Jiang L, Xu Y, Zhou Y, Shen Y, Xie W, et al. Synthesis and anticoagulant activity of sodium alginate sulfates. Carbohydr Polym 2011; 83 (4): 1797-1803.

18. Jiang H, Fang D, Hsiao BS, Chu B, Chen W. Optimization and characterization of dextran membranes prepared by electrospinning. Biomacromolecules 2004; 5 (2): 326-333. 


\section{1-396}

19. Rouillard AD, Berglund CM, Lee JY, Polacheck WJ, Tsui Y, Bonassar LJ, et al. Methods for photocrosslinking alginate hydrogel scaffolds with high cell viability. Tissue Eng Part C Methods 2010; 17 (2): 173-179.

20. Jeon O, Powell C, Solorio LD, Krebs MD, Alsberg E. Affinity-based growth factor delivery using biodegradable, photocrosslinked heparinalginate hydrogels. J Control Release 2011; 154 (3): 258-266.

21. Babavalian H, Latifi AM, Shokrgozar MA, Bonakdar S, Tebyanian H, Shakeri F. Cloning and expression of recombinant human plateletderived growth factor-BB in Pichia Pink. Cell Mol Biol (Noisy-le-grand) 2016; 62 (8): 45-51.

22. Shakeri F, Tebyanian H, Karami A, Babavalian H, Tahmasbi MH. Effect of Topical Phenytoin on Wound Healing. Trauma Mon 2017; 22 (5): e35488.

23. Babavalian H, Shokrgozar MA, Bonakdar S, Shakeri F, Tebyanian H. Healing Effects of Synthetic and Commercial Alginate Hydrogel Dressings on Wounds: A Comparative Study. Trauma Mon 2017; 22 (6): e38941.

24. Tebyanian H, Karami A, Motavallian E, Aslani J, Samadikuchaksaraei A, Arjmand B, et al. Histologic analyses of different concentrations of TritonX-100 and Sodium dodecyl sulfate detergent in lung decellularization. Cell Mol Biol (Noisy-le-grand) 2017 Aug 15; 63 (7): 46-51.

25. Tebyanian H, Karami A, Motavallian E, Aslani J, Samadikuchaksaraei A, Arjmand B, et al. A Comparative Study of Rat Lung Decellularization by Chemical Detergents for Lung Tissue Engineering. Open Access Maced J Med Sci 2017; 5 (7): 859-865.

26. Arunachalam KD, Subhashini S. Preliminary phytochemical investigation and wound healing activity of Myristica andamanica leaves in Swiss albino mice. J Med Plants Res 2011; 5 (7): 1095-1106.
27. Nishiyama T, Kii I, Kashima TG, Kikuchi Y, Ohazama A, Shimazaki M, et al. Delayed re-epithelialization in periostin-deficient mice during cutaneous wound healing. PLoS One 2011; 6 (4): e18410.

28. Chan RK, Liu PH, Pietramaggiori G, Ibrahim SI, Hechtman HB, Orgill DP. Effect of recombinant platelet-derived growth factor (Regranex $\left({ }^{\circledR}\right)$ on wound closure in genetically diabetic mice. J Burn Care Res 2006; 27 (2): 202-205.

29. Lin H, Chen B, Sun W, Zhao W, Zhao Y, Dai J. The effect of collagen-targeting platelet-derived growth factor on cellularization and vascularization of collagen scaffolds. Biomaterials 2006; 27 (33): 5708-5714.

30. Sun W, Sun W, Lin H, Sun W, Lin H, Xie H, et al. Collagen membranes loaded with collagen-binding human PDGF-BB accelerate wound healing in a rabbit dermal ischemic ulcer model. Growth Factors 2007; 25 (5): 309-318.

31. Drury JL, Dennis RG, Mooney DJ. The tensile properties of alginate hydrogels. Biomaterials 2004; 25 (16): 3187-3199.

32. Gu F, Amsden B, Neufeld R. Sustained delivery of vascular endothelial growth factor with alginate beads. J Control Release 2004; 96 (3): $463-472$.

33. Richardson TP, Peters MC, Ennett AB, Mooney DJ. Polymeric system for dual growth factor delivery. Nat Biotechnol 2001; 19 (11): 1029-1034.

34. Freeman I, Kedem A, Cohen S. The effect of sulfation of alginate hydrogels on the specific binding and controlled release of heparin-binding proteins. Biomaterials 2008; 29 (22): 3260-3268.

Received February 5, 2018. Accepted February 28, 2018. 